Kong. Res. J. 2(1) : 32-35, 2015

ISSN 2349-2694

Kongunadu Arts and Science College, Coimbatore.

\title{
EXISTENCE OF SOLUTIONS FOR NEUTRAL FUNCTIONAL VOLTERRA-FREDHOLM INTEGRODIFFERENTIAL EQUATIONS
}

\author{
Dhanalakshmi, S. and G. Deepa \\ Department of Mathematics (UA), Kongunadu Arts and Science College, Coimbatore. \\ *E.mail: dhana_bala16@yahoo.co.in
}

\section{ABSTRACT}

In this paper, we study the existence of mild solutions of nonlinear neutral functional VolterraFredholm integrodifferential equations with nonlocal conditions. The results are obtained by using fractional power of operators and Sadovskii's fixed point theorem.

Keywords: Integrodifferential equations, fixed point theorem, Banach space.

MSC subject classification : 45J05, 47H10, 46Bxx.

\section{INTRODUCTION}

Many phenomena in several branches have mathematical model in terms of differential equations. Differential equations are like a bridge which links mathematics and science with applications. It is a rightly considered as a language of sciences. Many branches of science have led to some kind of differential equations.

The study of integrodifferential equations has emerged in recent years as an independent branch of

modern research because of its connections to many applied fields such as elasticity, biology, epidemics and other branches of science and engineering. Neutral differential equations arises in many areas of applied mathematics and for this reasons this equations have received much attention in the last few decades.

The advantages of using nonlocal conditions is that measurable at more places can be incorporated to get better models. The nonlocal Cauchy problem for abstract evolution differential equation was first considered by Byszewski (Byszwski, 1991) Subsequently, several authors have investigated the problem for different types of nonlinear differential equations and integrodifferential equations including functional differential equations in Banach spaces (Balachandran, 1998; Byszwski and Acka, 1998; Balachandran and Park, 2001a, b; Fu and Ezzinbi, 2004).

In the past several years theorems about existence, uniqueness and stability of differential and functional differential abstract evolution Cauchy problem have been studied by Byszewski and Lakshmikantham (1990), Byszewski $(1997,1998)$,
Balachandran and Chandrasekaran (1996), Lin and Liu (1996) and Murugesu and Suguna (2010).

In this paper, we extend this problem to neutral functional Volterra-Fredholm type integro differential equations with nonlocal conditions and discuss the existence of solutions for nonlinear neutral functional Volterra-Fredholm integro differential equations with nonlocal conditions of the form

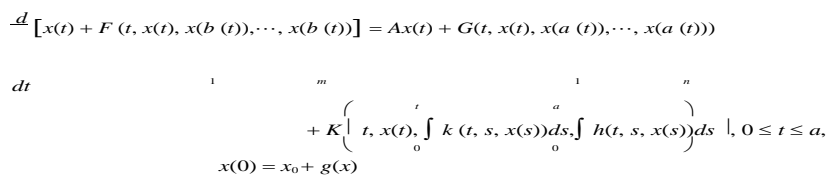

where -A generates an analytic semigroup and $\mathrm{F}, \mathrm{G}, \mathrm{K}, \mathrm{k}, \mathrm{h}$ are given functions to specifed later.

This paper has the following subsections. In section 2, we present some preliminary lemmas and definitions which will be used to prove our main results. In section 3, we present the existence of mild solution of the system (1) using Sadovskii's fixed point theorem (Sodovskii, 1967).

\section{PRELIMINARIES}

Throughout this work, let -A is the infinitesimal generator of a compact analytic semigroup of uniformly bounded linear operators $T(t)$ defined in the Banach spaces $X$. Let $0 \in \rho$ then define the fractional power $A^{\alpha}$, for $0 \leq \alpha \leq 1$, as a closed linear operator on its domain $D\left(A^{\alpha}\right)$ which is dense in $X$. Further, $\mathrm{D}(\mathrm{A})$ is a Banach space under the norm

$$
\|x\|_{\alpha}=\left\|A^{\alpha} x\right\|, x \in D\left(A^{\alpha}\right)
$$

Which we denote by $\mathrm{X}_{\alpha}$. Then for each $0<\alpha \leq 1$, $X_{\alpha} \rightarrow X_{\beta}$ for $0<\beta<\alpha \leq 1$ and the imbedding is 
compact whenever the resolvent operator of $\mathrm{A}$ is compact. We assume that

a) There is a $M \geq 1$ such that $\|T(t)\| \leq M$, for all $0 \leq t \leq a$.

b) For any a $>0$, there exists a positive constant $C_{\alpha}$ $\left\|A^{\alpha} T(t)\right\| \leq \frac{C_{\alpha}}{t^{\alpha}}, 0<t \leq a$

$$
\| A^{\beta} F(s, \underset{0}{x, x}, \cdots, x)-A_{m}^{\beta} F\left(s_{2}, \mathfrak{x}_{0}, \mathfrak{x}_{1},\right.
$$

for any $0 \leq \mathrm{s}_{1}, \mathrm{~s}_{2} \leq \mathrm{a}, \quad x_{i}, \bar{x}_{i} \in \mathrm{X}, \mathrm{i}=0,1, \ldots, \mathrm{m}$ and the inequality

$\left\|A^{\beta} F\left(t, x_{0}, x_{1}, \cdots, x_{m}\right)\right\| \leq L_{1}\left(\max \left\{\|x\|_{i} \|: i=0,1, \cdots, m\right\}+1\right)$

holds for any $\left(t, x_{0}, x_{1}, \ldots, x_{m}\right) \in[0, a] \times X^{m+1}$.

(H2) The function $G:[0, a] \times X^{n+1} \rightarrow X$ satisfies the following conditions :

(i) For each $t \in[0, a]$, the function $G(t,):. \times$ $\mathrm{X}^{\mathrm{n}+1} \rightarrow \mathrm{X}$ is continuous and for each $\left(\mathrm{x}_{0}, \mathrm{x}_{1}, \ldots\right.$, $\left.x_{n}\right) \in X^{n+1}$ the function $G\left(., x_{0}, x_{1}, \ldots, x_{n}\right):[0, a] \rightarrow X$ is strongly measurable.

(ii) For each positive number $n \in N$, there is a positive function $\phi_{\mathrm{n}} \in \mathrm{L}^{1}([0, \mathrm{a}])$ such that

$$
\begin{aligned}
\sup _{\left\|x_{0}\right\|, \cdots,\left\|x_{n}\right\| \leq n} \| & G\left(t, x_{\mathrm{O}}, x_{1}, \cdots, x_{n}\right) \| \leq \phi_{n}(t) \\
& \lim _{n \rightarrow \infty} \frac{1}{n} \int_{n}^{a} \phi_{n}(s) d s=\gamma<\infty
\end{aligned}
$$

(H3) The function $\mathrm{K}:[0, \mathrm{a}] \times \mathrm{X} \times \mathrm{X} \times \mathrm{X} \rightarrow \mathrm{X}$ satisfies the following conditions:

(i)

For each $t \in[0, a]$, the function $K(t$, ., .. .): $\mathrm{X}^{\times} \mathrm{X} \times \mathrm{X} \rightarrow \mathrm{X}$ and for each $\mathrm{X}, \mathrm{y}, \mathrm{z} \in \mathrm{X}, \mathrm{K}(., \mathrm{X}, \mathrm{y}, \mathrm{z})$ : $[0, a] \rightarrow X$ is strongly measurable.

(ii) For each positive number $\mathrm{n} \in \mathrm{N}$, there exists a positive function $\mathrm{q}_{\mathrm{n}} \in \mathrm{L}^{1}([0, \mathrm{a}])$ such that

$$
\begin{array}{r}
\sup _{\|x\| \leq n} \| K\left(s, x(s), \int_{0}^{s} k(s, \tau, x(\tau)) d \tau, \int h(s, \tau, x(\tau)) \| d \tau \mid \leq q_{n}(s)\right. \\
\text { and } \lim _{n \rightarrow \infty} \frac{1}{n} \int_{0}^{a} q_{n}(s) d s=\gamma_{1}<\infty
\end{array}
$$

(H4) a, b $\in \mathrm{C}([0, a] ;[0, a]), i=1, \ldots, n, j=1, \ldots, m$. $\mathrm{g} \in \mathrm{C}(\mathrm{E} ; \mathrm{X})$, here after $\mathrm{E}=$

$\mathrm{C}([0, \mathrm{a}] ; \mathrm{X})$ and $\mathrm{g}$ is completely continuous.

(H5) There exist positive constants $M_{3}$ and $M_{4}$ such that

$$
\|\mathrm{g}(\mathrm{x})\| \leq \mathrm{M}_{3}\|\mathrm{x}\|+\mathrm{M}_{4} \text { for every } \mathrm{x} \in \mathrm{E} .
$$

For our convenience, let us take

$$
F\left(0, x(0), x\left(b_{1}(0)\right), \ldots x\left(b_{m}(t)\right)\right)=0 .
$$

Let $\mathrm{M}_{0}=\left\|\mathrm{A}^{-\beta}\right\|$ and also assume the following hypotheses:

(H1) $\mathrm{F}:[0, \mathrm{a}] \times \mathrm{X}^{\mathrm{m}+1} \rightarrow \mathrm{X}$ is a continuous functions and there exists a $\beta \in(0,1)$ and $L_{,} L_{1}>0$ such that the

function $A^{\beta} \mathrm{F}$ satisfies the Lipschitz condition:

$$
\text { ) }
$$

DEFINITION : 2.1 (Pazy, 1983)

Let $\mathrm{X}$ be a Banach space, a one parameter family $T(t), 0 \leq t<+\infty$, of bounded linear operators from $\mathrm{X}$ to $\mathrm{X}$ is a semigroup of bounded linear operators on $\mathrm{X}$, if

(i) $\mathrm{T}(0)=\mathrm{I}$, where I is the identity operator on $\mathrm{X}$,

(ii) $\mathrm{T}(\mathrm{t}+\mathrm{s})=\mathrm{T}(\mathrm{t}) \mathrm{T}(\mathrm{s})$ for every $\mathrm{t}, \mathrm{s} \geq 0$, (the semigroup property)

A semigroup of bounded linear operator $T(t)$ is uniformly continuous if

$$
\lim _{t \downarrow 0}\|T(t)-I\|=0
$$

\section{THEOREM : 2.1. (SADOVSKII'S FIXED POINT THEOREM) (Sodovskii, 1967)}

Let $\psi$ be a condensing operator on a Banach space $\mathrm{X}$, that is $\psi$ is continuous and takes bounded sets into bounded sets and $\mu(\psi(B)) \leq \mu(B)$ for every bounded set $\mathrm{B}$ of $\mathrm{X}$ with $\mu(\mathrm{B})>0$. If $\psi(\mathrm{T}) \subset \mathrm{T}$ for a convex closed and bounded set $\gamma$ of $X$, then $\psi$ has a fixed point in $\mathrm{X}$.

\section{EXISTENCE OF MILD SOLUTION \\ DEFINITION 3.1.}

A continuous function $\mathrm{x}():.[0, \mathrm{a}] \rightarrow \mathrm{X}$ is said to be a mild solution of the Cauchy problem (1), if the function $\operatorname{AT}(t-s) F\left(s, x(s), x\left(b_{1}(s)\right), \ldots, x\left(b_{m}(s)\right)\right)$, $s \in[0, a)$ is integrable on $[0, a)$ and the integral equation is satisfied.

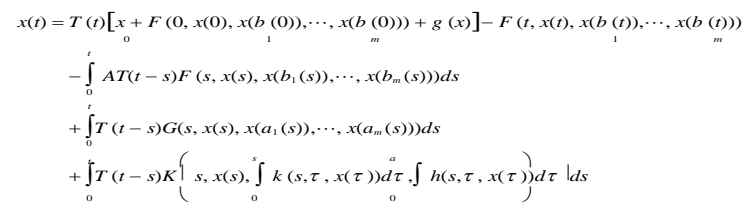

(4)

\section{THEOREM 3.1}

If the assumptions (H1) - (H5) are satisfied and $\mathrm{X}_{0} \in \mathrm{X}$, then the Cauchy problem (1) has a mild 
solution provided that

$$
\begin{aligned}
L:=L\left[M+{ }_{0}^{1} \frac{C}{{ }_{0}} \beta^{1-\beta}\right. & \left.a^{\beta}\right]<1
\end{aligned}
$$

Where $\mathrm{M}_{0}=\left\|\mathrm{A}^{-\beta}\right\|$.

Proof:

For the sake of brevity, we write that

$\left(t, x(t), x\left(b_{1}(t)\right), \ldots, x\left(b_{m}(t)\right)\right)=(t, v(t))$

and $\left(t, x(t), x\left(a_{1}(t)\right), \ldots, x\left(a_{m}(t)\right)\right)=(t, u(t))$.

Define the operator $\mathrm{Q}$ on $\mathrm{E}$ by the formula

$(Q x)(t)=T(t)\left[x_{0}+g(x)\right]-F(t, v(t))-\int^{t} A T(t-s) F(s, v(s)) d s+\int^{t} T(t-s) G(s, u(s)) d s$

$$
+\int_{0}^{t} T(t-s) K\left(s, x(s), \int_{0}^{s} k(s, \tau, x(\tau)) d \tau, \int_{0}^{a} h(s, \tau, x(\tau)) d \tau \mid d s\right.
$$

For positive integer $r$, let

$$
B_{r}=\{x \in E:\|x(t)\| \leq r, 0 \leq t \leq a\}
$$

then for each $\mathrm{r}$, $\mathrm{Br}$ is clearly a bounded closed convex set in E. Since by (2) and (3) the following relation holds:

$$
\begin{aligned}
\|A T(t-s) F(s, v(s))\| & \leq\left\|A^{1-\beta} T(t-s) A^{\beta} F(s, v(s))\right\| \\
& \leq \frac{C_{1-\beta} L}{(t-s)^{1-\beta} 1}(r+1)
\end{aligned}
$$

then from Bochners theorem (Marle, 1974) it follows that $\operatorname{AT}(\mathrm{t}-\mathrm{s}) \mathrm{F}(\mathrm{s}, \mathrm{v}(\mathrm{s}))$ is integrable on $[0, \mathrm{a}]$, so $\mathrm{Q}$ is well defined on $\mathrm{B}_{\mathrm{r}}$.

\section{Claim : there exists a positive integer $r$ such that} $\mathbf{Q B}_{\mathbf{r}} \subseteq \mathbf{B}_{\mathbf{r}}$ :

$r$, there is a it is not true, then for each positive integer $\left\|\mathrm{Qx}_{\mathrm{r}}(\mathrm{t})\right\|>\mathrm{r}$ for some $\mathrm{t}(\mathrm{r}) \in[\hat{\mathrm{f}}, \mathrm{a}]$, where $\mathrm{t}(\mathrm{r})^{\mathrm{r}}$ denotes

$t$ is dependent of $r$. However, on the other hand, we have

$$
\begin{aligned}
& r<\left\|Q x_{r}(t)\right\| \\
& =\| T(t)\left[x_{0}+g(x)\right]-F\left(t, v_{r}(t)\right)-\int_{0}^{t} A T(t-s) F\left(s, v_{r}(s)\right) d s+\int_{0}^{t} T(t-s) G\left(s, u_{r}(s)\right) d s \\
& +\int_{0}^{\prime} T(t-s) K\left(s, x(s), \int_{0} k(s, \tau, x(\tau)) d \tau, \int_{0}^{l} h(s, \tau, x(\tau)) d \tau\right)^{\prime} d s \| \\
& \leq M\|x d\|+M M n+M M+M L(r+1)+\frac{1}{\beta} C^{1-\beta} a^{\beta} L(r+1)+\prod_{0} \phi(s) d s+M \underset{0}{\operatorname{din}}(s) d s
\end{aligned}
$$

Dividing on bothsides by $\mathrm{r}$ and taking the limit as $\mathrm{r} \rightarrow$ $\infty$, we get,

$$
\left(\gamma+\gamma_{1}+M_{3}\right) M+M_{0} L_{1}+{ }_{\beta}^{1} C_{1-\beta} a^{\beta} L_{1} \geq 1
$$

This contradicts (6). Hence for some positive integer $\mathrm{r}, \mathrm{QB}_{\mathrm{r}} \subseteq \mathrm{B}_{\mathrm{r}}$.

\section{Next we will show that the operator $Q$ has a fixed point on $B_{r}$ :}

Let us decompose $\mathrm{Q}$ as $\mathrm{Q}=\mathrm{Q}_{1}+\mathrm{Q}_{2}$ where the operators $Q_{1}$ and $Q_{2}$ are defined on $B_{r}$ respectively by

$$
\begin{aligned}
\left(Q_{1} x\right)(t)= & -F(t, v(t))-\int_{0}^{t} A T(t-s) F(s, v(s)) d s \\
\left(Q_{2} x\right)(t)= & T(t)\left[x_{0}+g(x)\right]+\int_{0}^{t} T(t-s) G(s, u(s)) d s \\
& +\int_{0}^{t} T(t-s) K\left(s, x(s), \int_{0}^{s} k(s, \tau, x(\tau)) d \tau, \int_{0}^{a} h(s, \tau, x(\tau)) d \tau\right) d s
\end{aligned}
$$

for $0 \leq \mathrm{t} \leq \mathrm{a}$, and we will verify that $\mathrm{Q}_{1}$ is contraction and $\mathrm{Q}_{2}$ is a compact operator.

\section{Claim : $\mathbf{Q}_{\mathbf{1}}$ is a contraction}

Let $\mathrm{x}, \mathrm{x} \in \mathrm{B}$. Then for each $\mathrm{t} \in[0, \mathrm{a}]$ and by condition ( $\left.\mathrm{H}^{1}\right)$ and $(5)$, we have

$$
\begin{aligned}
& \left\|\left(Q_{1} x_{1}\right)(t)-\left(Q_{1} x_{2}\right)(t)\right\| \leq\left\|F\left(t, v_{1}(t)\right)-F\left(t, v_{2}(t)\right)\right\|\|\| \int_{C_{1-\beta}}^{t} A T(t-s)\left[F\left(s, v_{1}(s)\right)-F\left(s, v_{2}(s)\right) \| d s\right. \\
& \leq M_{0} L \sup _{0 \leq \leq \leq a}\left\|x_{1}(s)-x_{2}(s)\right\|+\int_{0} \frac{}{(t-s)^{1-\beta}} L d s \sup _{0 \leq s \leq a}\left\|x_{1}(s)-x_{2}(s)\right\| \\
& \leq L\left[M_{0}+{ }_{\beta}^{1} C_{1-\beta} a^{\beta}\right\rceil \text { sup } \mathbb{p}_{s}\left\|x_{1}(s)-x_{2}(s)\right\| \\
& \leq L_{0} \text { sup }\left\|x_{1}(s)-x_{2}(s)\right\|
\end{aligned}
$$

So by assumption $0<\mathrm{L}_{0}<1$, we see that $\mathrm{Q}_{1}$ is a contraction.

\section{Claim : $Q_{2}$ is compact}

To prove this we have to prove that $\mathrm{Q}_{2}$ is continuous on $\mathrm{B}_{\mathrm{r}}$.

Let $\left\{\mathrm{x}_{\mathrm{n}}\right\} \subseteq \mathrm{B}_{\mathrm{r}}$ with $\mathrm{x}_{\mathrm{n}} \rightarrow \mathrm{x}$ in $\mathrm{B}_{\mathrm{r}}$, then by (H2) (i), we have

$$
G\left(s, u_{n}(s)\right) \rightarrow G(s, u(s)), \quad n \rightarrow \infty
$$

$\mathrm{K}\left(\mathbf{t}, \mathbf{x}_{\mathrm{n}}(t), \int_{0}^{1} k\left(t, s, x_{n}(s)\right) d s, \int_{0}^{a} h\left(t, s, x_{n}(s)\right) d s\right\} \rightarrow \mathrm{K}\left(\mathbf{t}, \mathbf{x}(t), \int_{0}^{1} k(t, s, x(s)) d s, \int_{0}^{a} h(t, s, x(s)) d s \mid\right.$ as $n \rightarrow \infty$

Since $\left\|G\left(s, u_{n}(s)\right)-G(s, u(s))\right\| \leq 2 \phi_{n}(s)$,

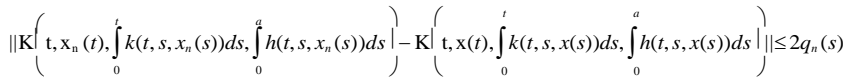
by the dominated convergence theorem, we have

$$
\begin{aligned}
& \|\underset{2 n}{Q x}-\underset{2}{Q x}\|=\sup _{0 \leq \leq \leq a}^{\|} T(t)[g(x) \underset{n}{n} g(x)]+\int_{0}^{t} T(t-s)[G(s, u(s))-G(s, u(s))] d s
\end{aligned}
$$

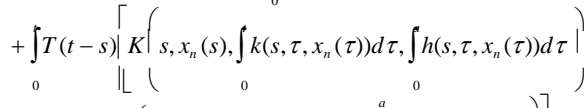

$$
\begin{aligned}
& -K^{(}\left|s, x(s) \int_{0}^{t} k(s, \tau, x(\tau)) d \tau, \int h(s, \tau, x(\tau)) d \tau\right| \mid d s \| \\
& \rightarrow 0 \text { as } \quad n \rightarrow \infty
\end{aligned}
$$

(i.e) $Q_{2}$ is continuous.

Next, we prove that $\left\{Q_{2} \mathrm{X}: \mathrm{x} \in \mathrm{B}_{\mathrm{r}}\right\}$ is a family of equicontinuous functions. To see this we fix $t_{1}>0$ and $t_{2}>t_{1}$ and $\varepsilon>0$ be enough small. Then 
$\left\|\left(Q_{2} x\right)\left(t_{2}\right)-\left(Q_{2} x\right)\left(t_{1}\right)\right\| \leq\left\|T\left(t_{2}\right)-T\left(t_{1}\right)\right\|\left\|\left|x_{0}+g(x)\left\|+\int\right\| \int^{n-k} T\left(t_{2}-s\right)-T\left(t_{1}-s\right)\|\|\right| G(s, u(s))\right\| d s$

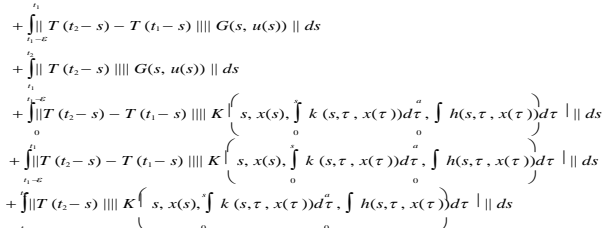

Note that $\|\mathrm{G}(\mathrm{s}, \mathrm{u}(\mathrm{s}))\| \leq \phi_{\mathrm{n}}(\mathrm{s})$ and $\phi_{\mathrm{n}}(\mathrm{s}) \in \mathrm{L}^{1}$, we see that $\left\|\mathrm{Q}_{2} \mathrm{x}\left(\mathrm{t}_{2}\right)-\mathrm{Q}_{2} \mathrm{x}\left(\mathrm{t}_{1}\right)\right\|$ tends to zero independently of $x \in B_{r}$ as $t_{2}-t_{1} \rightarrow 0$. Since the compactness of $T(t), t>0$ implies the continuity of $\mathrm{T}(\mathrm{t}), \mathrm{t}>0$ in $\mathrm{t}$ in the uniform operator topology.

We can prove that the function $\mathrm{Q}_{2} \mathrm{x}, \mathrm{x} \in \mathrm{B}_{\mathrm{r}}$ are equicontinuous at $t=0$. Hence $Q_{2}$ maps $B_{r}$ into a family of equicontinuous function.

\section{Claim : $V(t)=\left\{\left(Q_{2} x\right)(t): x \in B_{r}\right\}$ is relatively compact in $X$.} we define

Let $0<\mathrm{t} \leq \mathrm{a}$ be a fixed and $0<\varepsilon<\mathrm{t}$. For $\mathrm{x} \in \mathrm{B}_{\mathrm{r}}$,

$$
\begin{aligned}
\left(Q_{2, x}\right)(t)= & T(t)\left[x_{0}+g(x)\right]+\int_{0}^{t-\varepsilon} T(t-s) G(s, u(s)) d s \\
& +\int_{0}^{t-\varepsilon} T(t-s) K\left(s, x(s), \int_{0}^{s} k(s, \tau, x(\tau)) d \tau, \int_{0}^{a} h(s, \tau, x(\tau)) d \tau\right) d s \\
= & T(t)\left[x_{0}+g(x)\right]+T(\varepsilon) \int_{0}^{t-\varepsilon} T(t-\varepsilon-s) G(s, u(s)) d s \\
& +\left.T(\varepsilon) \int_{0}^{t-\varepsilon} T(t-\varepsilon-s) K\left(s, x(s), \int_{0}^{s} k(s, \tau, x(\tau)) d \tau, \int_{0}^{a} h(s, \tau, x(\tau)) d \tau\right)\right|_{d s}
\end{aligned}
$$

Then from compactness of $\mathrm{T}(\varepsilon), \varepsilon>0$ we obtain $\mathrm{V}_{\varepsilon}=\left\{\left(\mathrm{Q}_{2, \varepsilon} \mathrm{x}\right)(\mathrm{t}): \mathrm{x} \in \mathrm{B}_{\mathrm{r}}\right\}$ is relatively compact in $\mathrm{X}$ for every $\varepsilon, 0<\varepsilon<t$. Moreover, for every $\mathrm{x} \in \mathrm{B}_{\mathrm{r}}$, we have

$$
\begin{aligned}
& \left\|\left(Q_{2} x\right)(t)-\left(Q_{2, x} x\right)(t)\right\|=\int\|T(t-s) G(s, u(s))\| d s \\
& +\int_{t=\varepsilon}^{t} \| T(t-s) K\left(\left.\right|_{i}\left|s, x(s), \int_{0}^{t} k(s, \tau, x(\tau)) d \tau, \int h(s, \tau, x(\tau)) d \tau\right| \| d s\right. \\
& \leq M \int_{t=\varepsilon} g_{k}(s) d s+M \int_{\tau_{\varepsilon}} q_{r}(s) d s
\end{aligned}
$$

Therefore, there are relatively compact sets arbitrarily close to the set $V(t)$. Hence the set $V(t)$ is also relatively compact in $\mathrm{X}$.

Thus, by Arzela-Ascoli theorem, $Q_{2}$ is a compact operator. Those arguments enable us to conclude that $Q=Q_{1}+Q_{2}$ is a condensing map $B_{r}$, and by the Sadovskii's fixed point theorem there exist a fixed point $x($.$) for Q$ on $B_{r}$.Therefore, the Cauchy problem (1) has a mild solution, and the proof is completed.

\section{REFERENCES}

Balachandran, K. and J.Y. Park, (2001a). Existence of mild solution of a functional integrodifferential equation with nonlocal condition. Bull. Korean Math. Soc. 38: 175-182.
Balachandran, K. and J.Y. Park, (2001b). Existence of solution of second order nonlinear differential equations with nonlocal conditions in Banach spaces. Ind. J. Pure Appl. Math. 32: 1883-1892.

Balachandran, K. and M. Chandrasekaran, (1996). Existence of solutions of a delay differential equations with nonlocal condition. Ind. J. Pure Appl. Math. 27: 443-449.

Balachandran, K., (1998). Existence and uniqueness of mild and strong solutions of nonlinear integrodifferential equations with nonlocal conditions. Diff. Equ. Dyn. Sys. 6: 159-165.

Byszwski, L. and V. Lakshmikantham, (1990). Theorem about the existence and uniqueness of a solution of a nonlocal abstract cauchy problem in a Banach space. Appl. Anal. 40: 1119.

Byszwski, L. and Acka H., (1998). Existence of solutions of semilinear functional differential evolution nonlocal problems. Nonlinear Anal. 34: 65-72.

Byszwski, L., (1991). Theorems about the existence and uniqueness of solutions of semi linear evolution nonlocal Cauchy problem. J. Math. Anal. Appl. 162: 494-505.

Byszwski, L., (1997). On weak solutions of functional differential abstract nonlocal cauchy problem. Ann. Polonici. Math. 65: 163-170.

Byszwski, L., (1998). Application of properties of the right hand sides of evolution equation to an investigation of nonlocal evolution problem. Nonlinear Anal. 33: 413-426.

$\mathrm{Fu}, \mathrm{X}$. and K. Ezzinbi, (2004). Existence of solutions for neutral functional differential evolution equations with nonlocal conditions. Nonlinear Anal. 54: 215-227.

Lin, Y. and H. Liu, (1996). Semilinear integrodifferential equations with nonlocal cauchy problem. Nonlinear Anal. 26: 10231033.

Marle, C.M., (1974). Measures of Probabilities, Hermam, Paris,.

Murugesu, R. and S. Suguna, (2010). Existence of solutions for neutral functional integer differential equations. Tamkang J. Math. 41(2): 117-128.

Pazy, A., (1983). Semigroups of Linear Operators and Applications to Partial Differential Equations. Applied Mathemati- cal Sciences, Springer, New York, USA.

Sodovskii, B.M., (1967). On a fixed point principle. Fun. Anal. Appl. 1: 74-76. 